\title{
Detecting and Discriminating Shigella sonnei Using an Aptamer-Based Fluorescent Biosensor Platform
}

\author{
Myeong-Sub Song ${ }^{1,+}{ }^{\dagger}$, Simranjeet Singh Sekhon ${ }^{1,+}{ }^{+}$Woo-Ri Shin ${ }^{1,{ }^{\dagger}}$, Hyung Cheol Kim ${ }^{2}$, \\ Jiho Min ${ }^{3}$, Ji-Young Ahn ${ }^{1, *}$ and Yang-Hoon Kim ${ }^{1, *}$ \\ 1 School of Biological Sciences, Chungbuk National University, 1 Chungdae-Ro, Seowon-Gu, \\ Cheongju 28644, Korea; smst04@nate.com (M.-S.S.); simranjeet261@gmail.com(S.S.S.); \\ aomaya91@nate.com (W.-R.S.) \\ 2 Technology Transfer Center, Korea Research Institute of Bioscience \& Biotechnology, 125 Gwahak-Ro, \\ Yuseong-Gu, Daejeon 34141, Korea; hcgimm@gmail.com \\ 3 Department of Bioprocess Engineering, Chonbuk National University, 567 Baekje-daero, \\ Deokjin-Gu Jeonju, Jeonbuk 54896, Korea; jihomin@chonbuk.ac.kr \\ * Correspondence: jyahn@chungbuk.ac.kr (J.-Y.A.); kyh @chungbuk.ac.kr (Y.-H.K.); \\ Tel.: +82-43-261-2301 (J.-Y.A.); +82-43-261-3575 (Y.-H.K) \\ + These authors contributed equally to this work.
}

Academic Editor: Harri Lönnberg

Received: 19 April 2017; Accepted: 14 May 2017; Published: 17 May 2017

\begin{abstract}
In this paper, a Whole-Bacteria SELEX (WB-SELEX) strategy was adopted to isolate specific aptamers against Shigella sonnei. Real-time PCR amplification and post-SELEX experiment revealed that the selected aptmers possessed a high binding affinity and specificity for $S$. sonnei. Of the 21 aptamers tested, the $\mathrm{C}(\mathrm{t})$ values of the SS-3 and SS-4 aptamers $(\mathrm{Ct}=13.89$ and $\mathrm{Ct}=12.23$, respectively) had the lowest value compared to other aptamer candidates. The SS-3 and SS-4 aptamers also displayed a binding affinity $\left(K_{D}\right)$ of $39.32 \pm 5.02 \mathrm{nM}$ and $15.89 \pm 1.77 \mathrm{nM}$, respectively. An aptamer-based fluorescent biosensor assay was designed to detect and discriminate $S$. sonnei cells using a sandwich complex pair of SS-3 and SS-4. The detection of S. sonnei by the aptamer based fluorescent biosensor platform consisted of three elements: (1) 5'amine-SS-4 modification in a 96-well type microtiter plate surface ( $N$-oxysuccinimide, NOS) as capture probes; (2) the incubation with S. sonnei and test microbes in functionalized 96 assay wells in parallel; (3) the readout of fluorescent activity using a Cy5-labeled SS-3 aptamer as the detector. Our platform showed a significant ability to detect and discriminate $S$. sonnei from other enteric species such as E. coli, Salmonella typhimurium and other Shigella species (S. flexneri, S. boydii). In this study, we demonstrated the feasibility of an aptamer sensor platform to detect $S$. sonnei in a variety of foods and pave the way for its use in diagnosing shigellosis through multiple, portable designs.
\end{abstract}

Keywords: shigellosis; aptamer-based fluorescent biosensor; whole cell-SELEX; DNA aptamer

\section{Introduction}

The Shigella species of bacteria is the causative agent of shigellosis, also known as bacillary dysentery, which has symptoms ranging from dysentery, tenesmus, and abdominal cramps to diarrhea with blood and mucous [1]. It is estimated that more than 165 million cases are reported annually and approximately one million people die from shigellosis worldwide each year [2]. The Shigella genus is comprised of four species: Shigella dysenteriae, Shigella flexneri, Shigella boydii, and Shigella sonnei. S. flexneri is the most common species in developing countries whereas $S$. sonnei is the predominant species in industrialized countries [3,4]. Several reports have shown that most shigellosis outbreaks are closely related to an influx of people with a history of international travel, which is difficult to 
control due to their low infectious dose [5,6]. Moreover, since shigellosis tends to spread very rapidly by mass feeding in closed and concentrated groups, such as army barracks and schools [7], there is an urgent need for rapid, simple, and specific detection methods for shigellosis.

The conventional plate-based method for detecting foodborne pathogens has the limitations of being time-consuming and requiring selective media. Shigella species are fastidious bacteria that survive poorly outside the human body, therefore plate-based methods can detect only a small fraction of shigellosis cases [8]. To overcome the limitations of the conventional culture method, rapid molecular diagnostic methods like real-time PCR [9] and PCR-ELISA [10] have been introduced. Although molecular detection methods using virulence genes are highly sensitive, they still have several limitations: (1) non-specific amplification and primer dimerization, (2) failure to recognize bacteria serotypes and antibiotic resistance (3) requirement of specialized lab equipment such as gel-electrophoresis apparatuses and/or antibodies.

More recently, many biosensor approaches for detecting pathogenic bacteria have been investigated, and this biosensor platform offers the advantage of label-free, real-time, and visible detection [11,12]. As an ideal element for recognition, specific aptamers in bacteria have been increasingly targeted over the use of antibodies in a variety of analytic technologies. Several efforts have been reported, such as the Surface Plasmon Resonance (SPR) analysis [13], aptamer-based lateral flow dipstick tests [14], cross-linked hydrogels [15], aptablotting assay [16].

An aptamer is an artificial single-stranded oligonucleotide that can be specifically isolated through the Systematic Evolution of Ligands by exponential enrichment (SELEX) process and capable of recognizing a variety of targets in systemically designed detection platforms [17-20]. Some studies have reported ingenious efforts to combine the recognition ability of aptamers with advanced nanomaterials in the field of drug delivery and targeted therapeutics because of their strong stability under a wide range of temperature, $\mathrm{pH}$ and in-vivo/ex-vivo conditions [21,22]. In a recent study, a sandwich-based detection method using dual aptamers to recognize S. sonnei has also been reported [23]. Herein, we present a specific aptamer sandwich pair against $S$. sonnei in a fluorescence detection platform. We have carried out a Whole-Bacteria SELEX process to obtain single stranded DNA aptamers with high affinity and specificity for $S$. sonnei. Aptamers were selected using whole live $S$. sonnei cells as targets, while E. coli cells were used as a negative control. After 10 rounds of selection, the aptamer pair, SS-3 and SS-4 were chosen and designed as the detection and capture probe in our aptamer-based fluorescent biosensor platform. Our platform allow for the rapid and sensitive discrimination between S. sonnei and other enteric bacteria, such as E. coli, Salmonella typhimurium and other Shigella species (S. flexneri, S. boydii).

\section{Results}

\subsection{Whole Bacteria Cell SELEX for the Isolation of S. sonnei Specific DNA Aptamers}

The randomized oligonucleotide aptamer library, containing about $10^{16}$ diverse sequences, was initially introduced by incubating cultured live S. sonnei cells. In each round of selection against S. sonnei, the concentration of bound DNA species was increased to further drive selection toward a high-affinity and high-specificity aptamer pool. The initial oligonucleotide library consisted of a 40 nucelotide randomized region $\left(\mathrm{N}_{40}\right)$, and primer binding sites were chemically synthesized. After 10 rounds of SELEX, nanodrop spectrophotometer analysis was performed to monitor and assess the binding affinity of aptamer sub-pools to $S$. sonnei. To eliminate non-specific and weakly binding DNA aptamers, we incorporated a negative-selection step using E. coli cells after the 6th selection round. It is important to note that gram-negative E. coli is closely related to Shigella spp. and discriminating Shigella from E. coli is clinically necessary, since Shigella is a source of infectious diarrhea which can cause a worldwide outbreak [24,25]. Genetically, the chromosomal structure of S. sonnei has the same replication origin and terminus as E. coli [26,27]; this means not only they evolutionarily related, but they most likely utilize the same cellular machinery in both DNA replication and in the 
gene expression/regulation processes for extracellular structure. As described in Figure 1, during the SELEX process, non-specific and/or weakly binding aptamer candidates were efficiently excluded using the negative count-partner, E. coli, in the 6th round. Thus the selected cell-specific aptamers can specifically recognize the outer-cellular targets of $S$. sonnei and can be used as high affinity binding probes in the detection platform. To determine if the DNA aptamer against $S$. sonnei cells would be enriched during the SELEX process, the amount of recovered ssDNA from each selection round was analyzed. The eluted concentration was highest in the 8th round after the negative selection (6th) round and 7th selection round. These results indicate that the ssDNA aptamer candidates were successfully enriched for whole live bacterial cells during the process. A decrease in ssDNA concentration was also observed after the negative selection round due to the removal of unbound ssDNA. We therefore determined that the 8th selection round is the optimal state to isolate appropriate aptamer candidates.

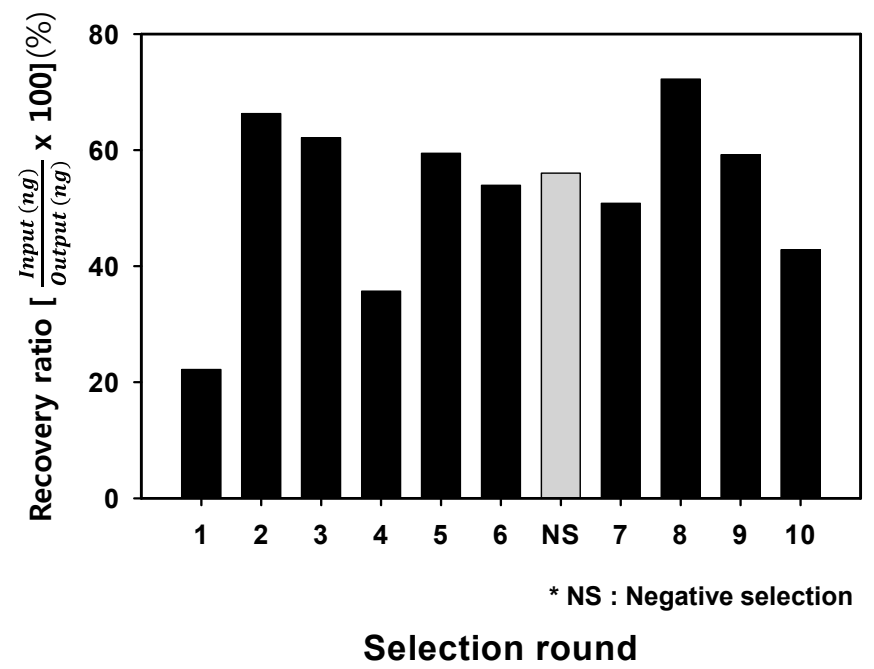

Figure 1. Measurement of eluted aptamers during the SELEX processes. The ssDNA was obtained from each selection round. The recovery ratio was calculated using the amount of input and output DNA concentration through the 10th round of the SELEX processes. The negative selection round (NS) was performed after round 6 to prevent enrichment of non-specific binding aptamers.

\subsection{Analysis of Optimal Enrichment States for Isolating Aptamers}

The retrieval concentration pattern showed that the overall tendency to increase with each selection round progressed until the 8th round, after which it decreased. This implies that the cell specific aptamers were saturated and enriched during the SELEX processes. To further demonstrate the difference in binding ability round by round, we employed real-time PCR (RT-PCR), which proved valuable in validating the optimal state of DNA aptamer enrichment [20].

Potential aptamer pools collected from the initial ( $0 \mathrm{R}), 7$ th, 8 th, and 9th round were incubated with $S$. sonnei cells $\left(1.2 \times 10^{8} \mathrm{cfu} / \mathrm{mL}\right)$ in parallel for $1 \mathrm{~h}$. After washing three times, bound DNA was recollected. The recovered DNA samples were each amplified using an optimized RT-PCR cycle and the fluorescent signal was monitored by the MiniOpticon ${ }^{\mathrm{TM}}$ Real-time (RT)-PCR fluorescent signal detection system (Bio-Rad, CA, USA). The PCR experiment was independently conducted in triplicate and data were analyzed to obtain the average $C(t)$ values (Figure S1). The samples from the 8th round SELEX showed a lower $C(t)(p<0.001), C(t)=13.66$, as compared to other samples. The $C(t)$ for the 7 th and 9th rounds were 14.33 and 13.85, respectively. Based on these RT-PCR experiments, it was clear that the sample collected from the 8th round pool did show a higher amount of DNA aptamers for S. sonnei cells.

To isolate potential aptamers that recognize whole live S. sonnei cells, the DNA aptamer pool from the 8 th selection round was cloned, followed by the sequencing of 40 clones. By multiple sequence 
alignment using the ClustalX software program, a total of 21 aptamers (SS-1 to SS-21) with intact random sequences were obtained (Table S1).

\subsection{Binding Test of Aptamer Candidates by Post-SELEX}

The binding value for each apamers was determined by analyzing the SPR and $\mathrm{C}(\mathrm{t})$ value using RT-PCR after the post-SELEX experiment (Figure S1 and Table 1). For the $\mathrm{C}(\mathrm{t})$ value analysis, 180 pmol of each aptamer was incubated with $S$. sonnei cells $\left(4 \times 10^{8} \mathrm{cfu} / \mathrm{mL}\right)$ at $4{ }^{\circ} \mathrm{C}$ for $1 \mathrm{~h}$ in $200 \mu \mathrm{L}$. After washing, each aptamer-cells complex was prepared for PCR amplification. The precipitated aptamers with cells were recovered and mixed with primers and PCR mixtures. Expecting that all of the unhybridized or weakened DNA aptamers would be eliminated by washing three times with $500 \mu \mathrm{L}$ of TE buffer in this post-SELEX step, the $\mathrm{C}(\mathrm{t})$ value by RT-PCR analysis showed which aptamer could tightly bind to their target cells. Of the 21 aptamers tested, results in Figure 2 show that the $C(t)$ value of SS-3 and SS-4 aptamers was significantly lower $(C t=13.89$ and $C t=12.23$, respectively) than that obtained when the other aptamers were used $(\mathrm{Ct}>14)$. It is understandable that most of the 19 aptamers were weakly bound to S. sonnei cells, whereas SS-3 and SS-4 aptamers showed tight binding to their target cells. Binding ability (SS-3 and SS-4) for detecting whole live S. sonnei was then evaluated by performing SPR assays with a nonspecific control (random aptamer pool) at the same cell concentration. The biotinylated ssDNA aptamers (SS-3, SS-4 and the random aptamer pool) were coupled to the streptavidin sensor chip. The whole cell binding experiment was carried out in an activation buffer (HBS-EP) solution. It was confirmed that significant aptamer binding signal came from the recognition of $S$. sonnei. No significant binding was observed in the random aptamer pool. Aptamer repeatability was also tested by removing target cells from the aptamer immobilized layer using $\mathrm{NaCl}$ treatment and then reinjecting the target cells. The complete detection cycle was observed without altering the aptamer's binding property during the consecutive interactions with S. sonnei $\left(4 \times 10^{8} \mathrm{cfu} / \mathrm{mL}\right)$ and regeneration in flow (Figure 3$)$. The SS-3 and SS-4 aptamers displayed binding affinity $\left(K_{D}\right)$ of $39.32 \pm 5.02 \mathrm{nM}$ and $15.89 \pm 1.77 \mathrm{nM}$, respectively (Table 1 ). In order to compare the secondary structures of the two aptamers, SS-3 and SS-4, we used the web-based tool Mfold (http:/ / mfold.bioinfo.rpi.edu/cgi-bin/dna-form1.cgi). The results of both the sequences and secondary structures of the SS-3 and SS-4 aptamers are given in detail in Table 1. No consensus was found between SS-3 and SS-4.

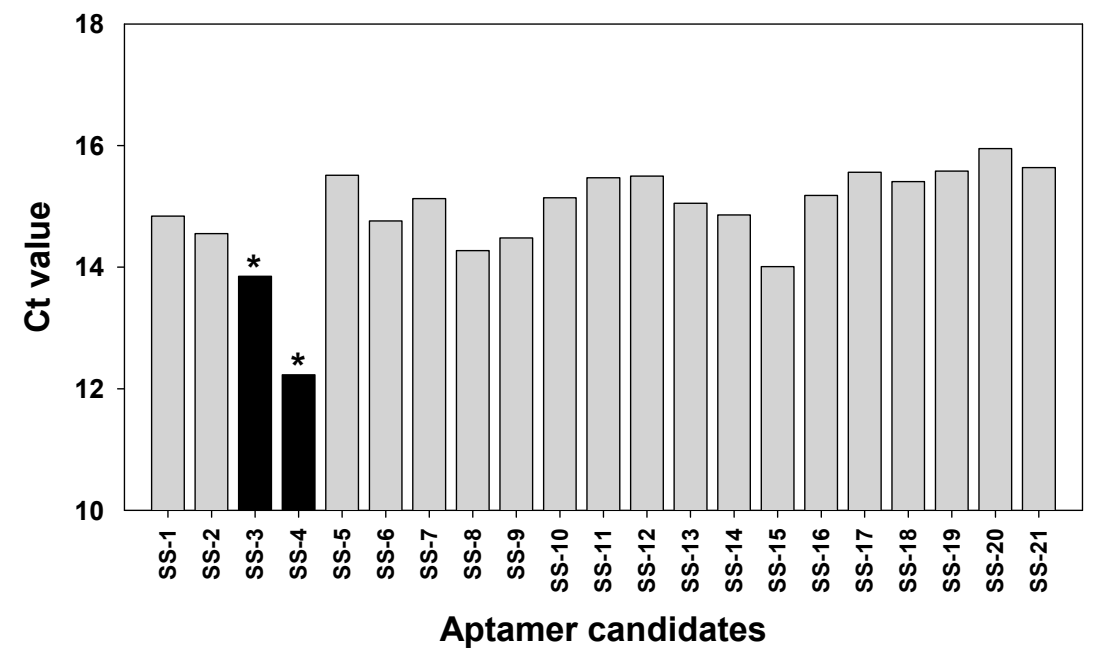

Figure 2. Characterization of binding aptamers. Twenty-one aptamer candidates (SS-1 to SS-21) were analyzed by a post-SELEX method as described in Materials and Method. After incubation with $S$. sonnei cells, aptamers were retrieved. The eluents were amplified by RT-PCR and the average $C(t)$ values were analyzed in triplicate individual experiments. *: $\mathrm{C}(\mathrm{t})$ value $<14$. 


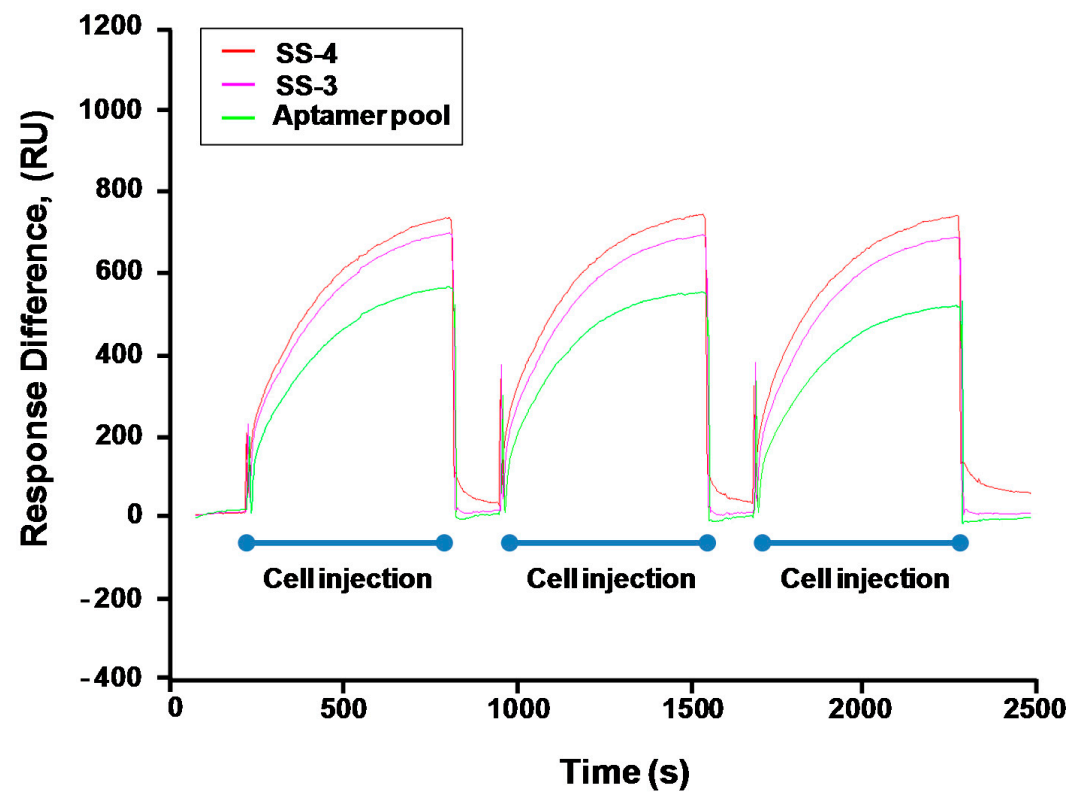

Figure 3. Repeatability of aptamer: Aptamer reusability was tested by removing target cells from the aptamer immobilized layer using $\mathrm{NaCl}$ treatment and injecting right after the target cells. The aptamer binding property was not altered during the consecutive interaction cycles with $S$. sonnei $\left(4 \times 10^{8} \mathrm{cfu} / \mathrm{mL}\right)$ and regeneration in flow.

Table 1. Sequence of the isolated aptamer candidates.

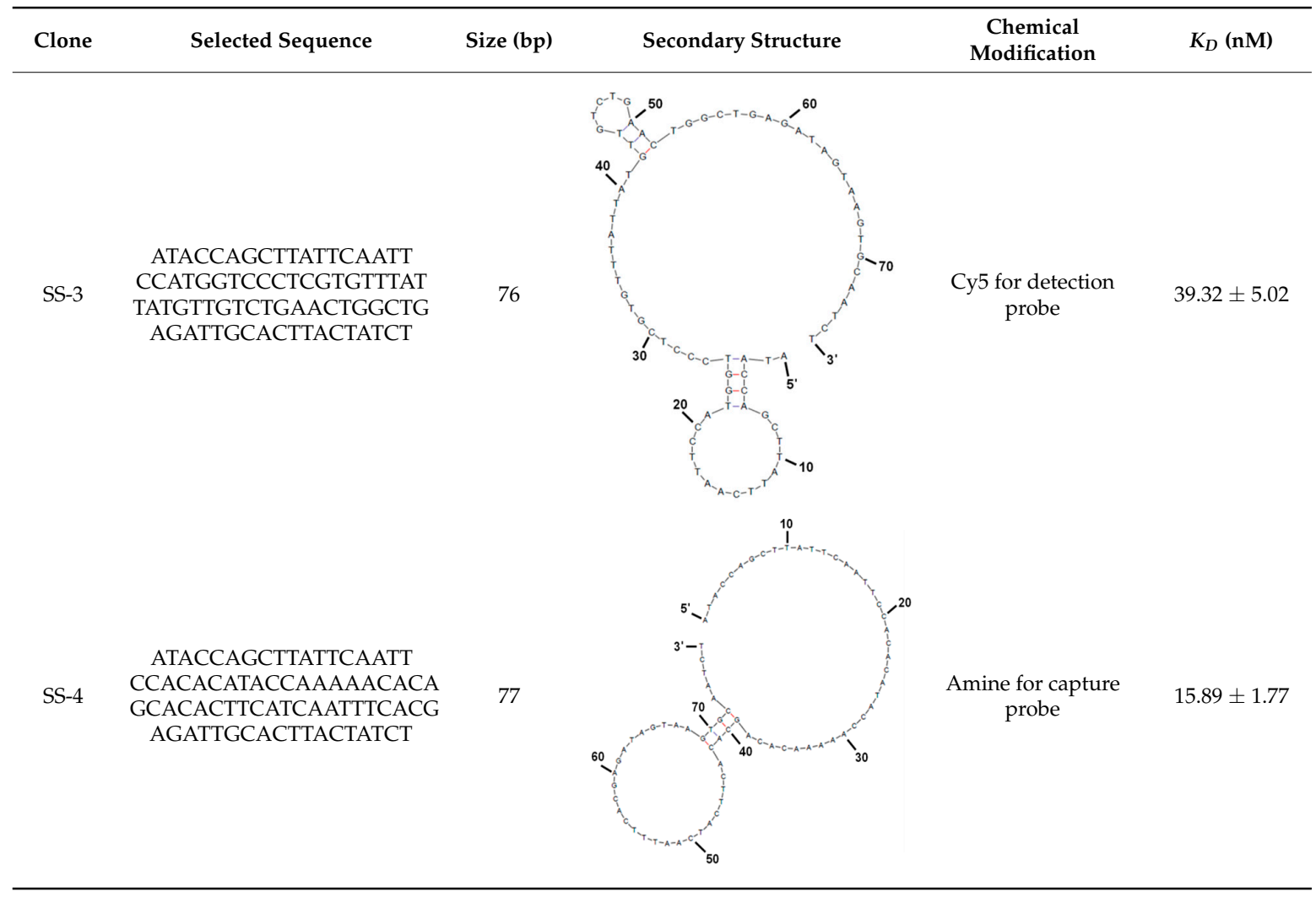




\subsection{Detection and Discrimination of S. sonnei by Aptamer-Based Fluorescent Biosensor}

An aptamer-based fluorescent biosensor assay has been designed to detect and discriminate S. sonnei cells using a sandwich complex pair of SS-3 and SS-4. The principle of the biosensor strategy is illustrated in Figure 4. First, a carboxyl group modified 96-well type microtiter plate surface (NOS chip surface) was activated using 5'amine-SS-4 as the capture probes. S. sonnei and test microbes were then added to the functionalized well in parallel. Finally, the capture signal with fluorescence activity was reported by SS-3, the Cy5-labeled detection aptamer. In detail, the surfaces were first functionalized by using an aptamer solution with a concentration of $1 \mu \mathrm{M}(100 \mathrm{pmol}$ per $100 \mu \mathrm{L}$ of treatment volume) per well. If fact, although $1 \mu \mathrm{M}$ of the $5^{\prime}$ amine modified aptamer (SS-4) was used to covalently bound to the NOS-well surface, it was necessary to determine the actual amount of apatmer bound to each well of the microplate.

Sensor wells were then challenged with different bacterial cells (S. flexneri (KCTC 2517), S. boydii (KCTC 22528), E. coli (KCTC 1116), and S. typhimurium (KCTC 2053)). The strains were diluted in commercially available bottled water $\left(4 \times 10^{8} \mathrm{cfu}\right.$ per $\left.\mathrm{mL}\right)$. After incubating with 500 pmol of the Cy5-labeled SS-3, the fluorescence intensity detected from the microtiter plate was recorded at $\lambda \mathrm{ex}=646 \mathrm{~nm}$ and $\lambda \mathrm{em}=662 \mathrm{~nm}$. We observed significantly increased fluorescence intensity value only in the S. sonnei cells as shown in Figure 5. Supplementary Figure S2 shows the intensity of the fluorescent signal, which represents the aptamer SS-4/target cells/SS-3-cy5 complex. A linear dynamic relationship between the aptamer sensor and the cells (within the range of $10^{3}-10^{6}$ cells per $\mathrm{mL}$ ) is seen, and at over $10^{7}$ cells binding signals were saturated.

As control experiments, the activated and functionalized surface by capture aptamer (control_A), the carboxyl group coated microtiter plate surface (control_B), and aptamer-aptamer binding without bacteria cells (control_C) were compared simultaneously. S. sonnei detection by aptamer sandwich pairs in our aptamer-based fluorescent biosensor platform was clearly distinguishable from the controls (A to C), nutrient broth (LB), buffer solution (TBS), and DW.

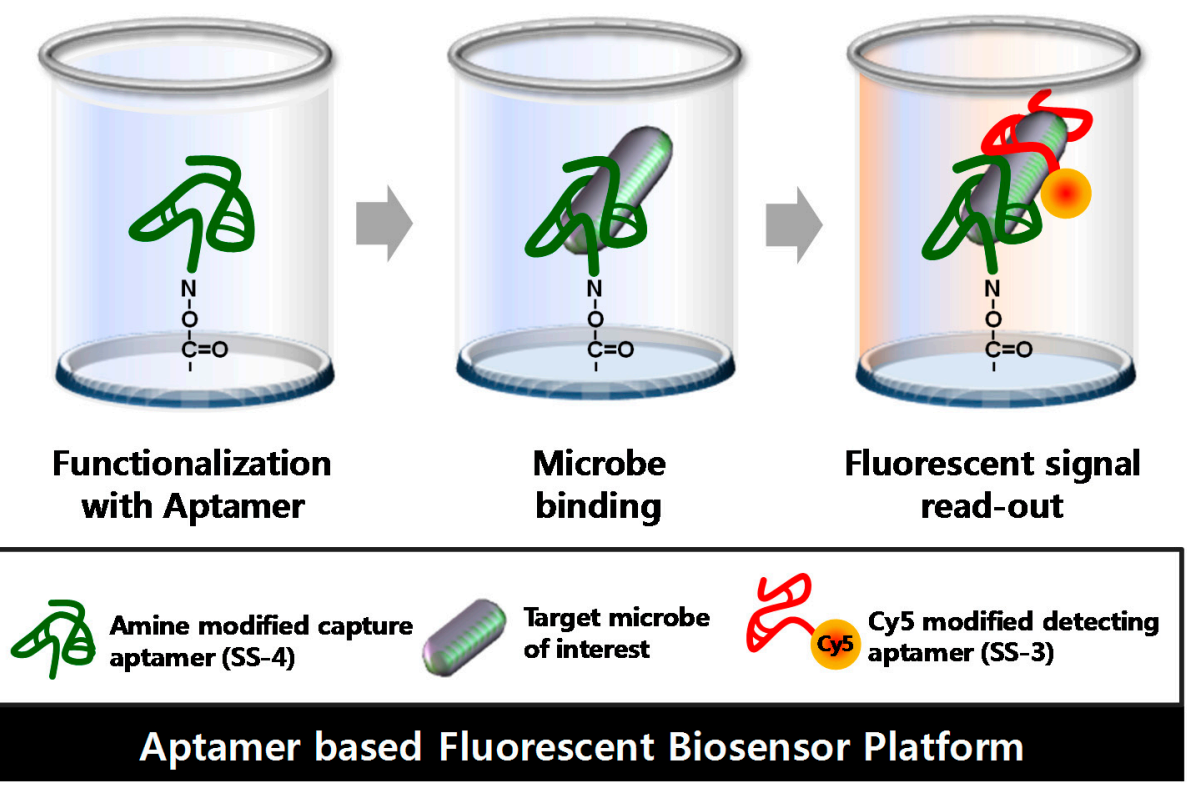

Figure 4. Principle of S. sonnei detection using an aptamer-based fluorescent biosensor platform. An aptamer-based fluorescent biosensor platform comprised of three major factors: the functionalized surface by the capture aptamer (SS-4), target binding, and signal read-out by the Cy5-labelled detection aptamer (SS-3). 


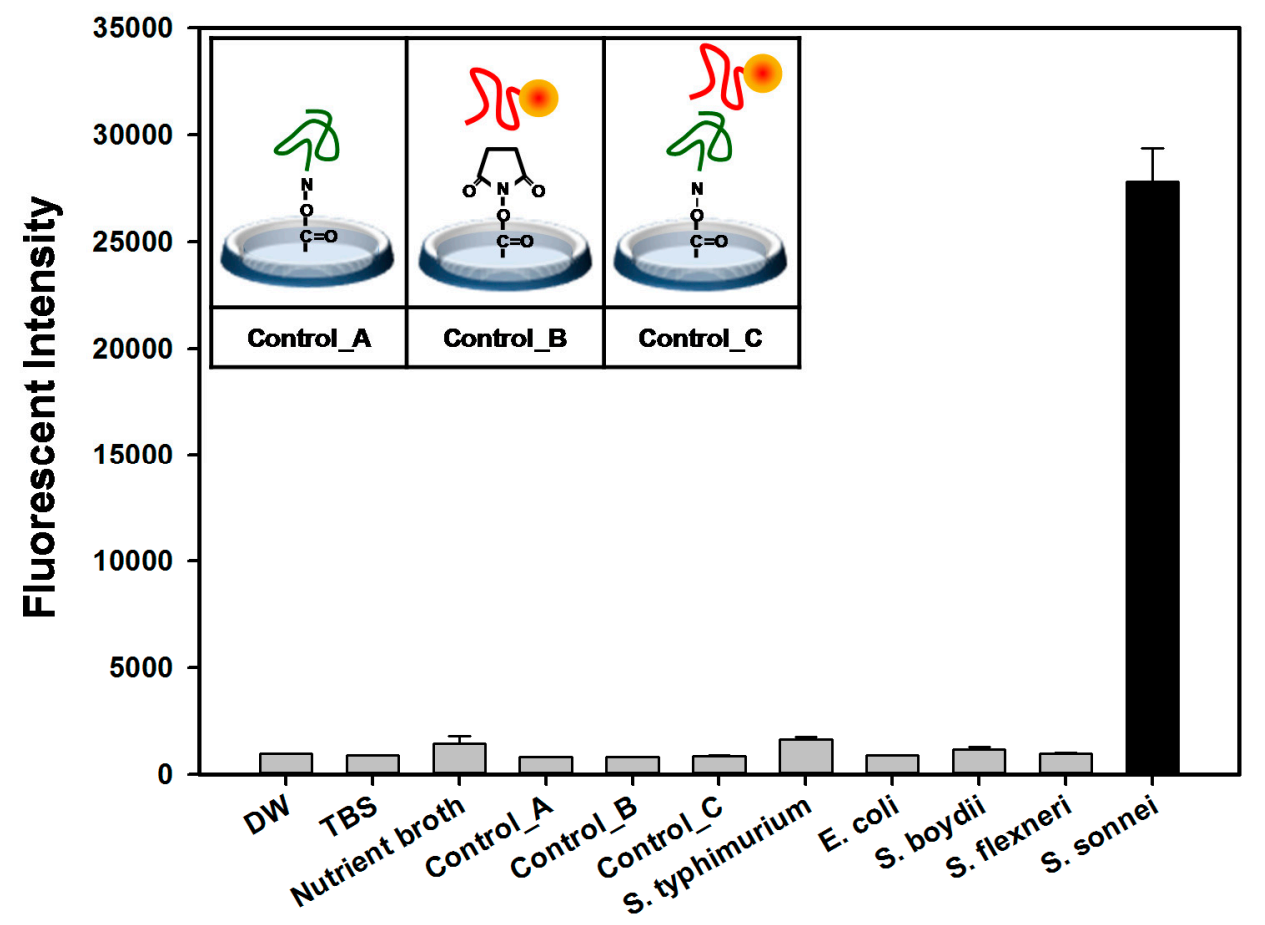

Figure 5. Sandwich binding activity of S. sonnei. Fluorescence intensity with three controls (A: basal signals of the plate surface with the detection aptamer; B: signals without the capture aptamer; C: signals without the target cells in sandwich complex) was determined by using an aptamer based fluorescent biosensor assay.

Concentration dependent aptamer binding activity against $S$. sonnei was also tested on the sandwich sensor assay. Results in Figure 6 show that SS-3 binds to target cells in a concentrationdependent manner.

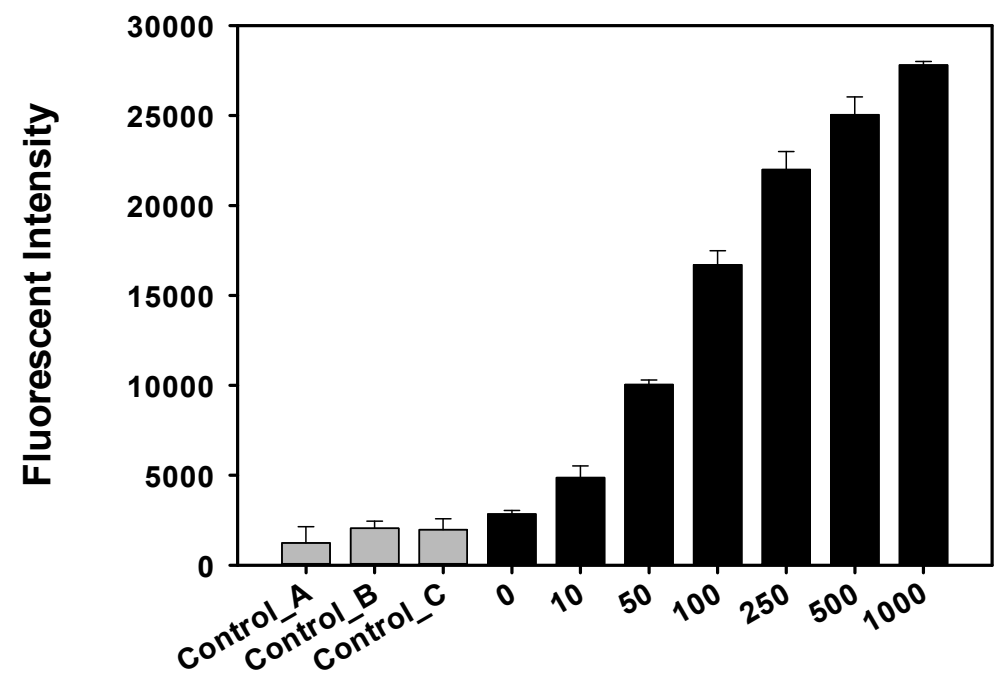

SS-3 concentration (nM)

Figure 6. Sandwich binding activity of S. sonnei. Various concentrations of SS-3 were used. Fluorescence intensity with three controls (A: basal signals of the plate surface with the detection aptamer, B: signals without the capture aptamer, C: signals without the target cells in sandwich complex) was determined by using an aptamer- based fluorescent biosensor assay. 
These results indicated that competitive binding between SS-3 and SS-4 was not observed. Therefore, SS-3 and SS-4 are assumed to have different binding sites on the surface of S. sonnei. Supplementary Figure S2 shows the reliable and sensitive quantification of $S$. sonnei with a linear dynamic range of $10^{3}-10^{6}$ cells $/ \mathrm{mL}\left(\mathrm{R}^{2}=0.97\right)$, and a limit of detection of $10^{3}$ cells per $\mathrm{mL}$.

\section{Discussion}

Many types of $S$. sonnei detection methods have been developed over the last several decades, including immunosorbent assays (ELISA) and polymerase chain reaction (PCR) amplification analysis. However, ELISA and PCR-based amplification assays have a high risk of false-positives and false-negatives due to the inhibition by the cellular component itself and the clinical sample matrix. Moreover, ELISA requires labeled primary and/or secondary antibodies. There is considerable cross-reactivity in the use of antibodies which can recognize Gram-negative bacteria serotype $\mathrm{O}$ antigens since many $\mathrm{O}$ groups of $E$. coli are cross-reactive or identical with specific $\mathrm{O}$ regions of Shigella [28]. In this paper, an aptamer-based fluorescent biosensor platform was developed and evaluated for critical discrimination of $S$. sonnei cells from other enteric pathogens. The biosensor platform consisted of a molecular recognition element pair, amine-modified capture aptamer (SS-4) and fluorescent labeled Cy5-detecting aptamer (SS-3). These aptamers were efficiently selected by Whole Bacteria-SELEX (WB-SELEX). A key aspect of the SELEX experiment was the recognition of the optimal enriched round during the process.

In fact, SELEX process can be modified in a variety ways to improve the aptamer specificity including the participation of counter-/negative selection and the incorporation of stringent buffer conditions during each round [29]. Here we used a post-SELEX procedure and real-time PCR analysis to obtain a highly efficient selection of aptamers. The counter-partner bacteria E. coli, which is closely related to $S$. sonnei in evolution, was used for negative selection of non-specific aptamers. A significantly increased intensity of fluorescence was observed for only the $S$. sonnei cells, not $S$. flexneri or S. boydii.

Our aptamer sensor platform therefore exhibits a high specificity that may be due to the formation of secondary and tertiary structures against a certain extracellular component of $S$. sonnei. In particular, the aptamer provides a clear modification mechanism for integrating it onto a solid surface within the sensor platform. This allows a specific and unique orientation to detect targets when anchored to the surface. This approach has the potential to be utilized in a direct sample-to-result detection of pathogens in multiple, portable designs. It may also represent an important development in diagnostics and therapeutics involving other pathogens by simply substituting aptamers.

\section{Materials and Methods}

\subsection{Bacterial Strains and Cell Maintenance}

Shigella sonnei (KCTC 2518), Shigella flexneri (KCTC 2517), Shigella boydii (KCTC 22528), Escherichia coli (KCTC 1116), and Salmonella typhimurium (KCTC 2053) were obtained from the Korean Collection for Type Cultures (KCTC, Jeongeup-si, South Korea). The media for bacterial culture were: nutrient broth (BD Difco, Oxford, UK) for S. sonnei, S. flexneri, and S. typhimurium; Tryptic Soy Broth (BD Difco) for S. boydii; Luria-Bertani media (LB; BD Difco) for Escherichia coli. All bacteria were cultured at $37^{\circ} \mathrm{C}$ with shaking at $180 \mathrm{rpm}$.

\subsection{Whole SELEX Pprocedure}

The initial oligonucleotide library consists of a 40-nucleotide randomized region $\left(\mathrm{N}_{40}\right)$ and primer binding sites was chemically synthesized (Bioneer, Daejeon, Korea): 5'-ATA CCA GCT TAT TCA ATT $-\mathrm{N}_{40}$ - AGA TAG TAA GTG CAA TCT-3'. The random library was amplified by PCR using forward primer (5'-ATA CCA GCT TAT TCA ATT) and biotinylated reverse primer (5'-AGA TTG CAC TTA CTA TCT-3') during the selection process. PCR product was purified using the QIAquick PCR purification kit (Qiagen, Germantown, MD, USA) and single-stranded DNA (ssDNA) was generated 
by removing the bionitylated double-stranded DNA (dsDNA) using streptavidin agarose resin (Pierce, WA, USA). The resulting ssDNA was confirmed by comparison with double-stranded DNA (PCR product) on $10 \%$ polyacrylamide gel containing $0.5 X$ TBE buffer (Thermo Fisher Scientific Korea, Seoul, Korea). The ssDNA library ( $800 \mathrm{pmol})$ was then heat denatured for $5 \mathrm{~min}$ at $85^{\circ} \mathrm{C}$ and renatured for $2 \mathrm{~h}$ at room temperature (RT) to allow the formation of a stable structure.

The subculture of $S$. sonnei $\left(4 \times 10^{8} \mathrm{cfu} / \mathrm{mL}\right)$ was washed with TBS buffer $(10 \mathrm{mM}$ Tris- $\mathrm{HCl}$, $0.85 \% \mathrm{NaCl}, \mathrm{pH} 8.0)$ and cell pellets were incubated with structured ssDNA library (800 pmol) at $4{ }^{\circ} \mathrm{C}$ with gentle shaking for $1 \mathrm{~h}$ in $200 \mu \mathrm{L}$ nutrient broth. Following incubation, the mixture was centrifuged (3500 rpm at $4{ }^{\circ} \mathrm{C}$ for $5 \mathrm{~min}$ ) and washed with TBS buffer to remove any unbound or weakly bound aptamers. To elute cell-bound aptamers, the cells were re-suspended in the TE buffer $(10 \mathrm{mM}$ Tris- $\mathrm{HCl}$, $1 \mathrm{mM}$ EDTA, pH 8.0), heated at $85^{\circ} \mathrm{C}$ for $5 \mathrm{~min}$, snap-cooled on ice, and centrifuged. The eluted ssDNA in supernatant was recovered by phenol/chloroform extraction and ethanol precipitation and then used as the template DNA for the next round of selection. The resulting purified aptamers were run on a $1 \%$ agarose gel to confirm correct DNA size. To increase the specificity of aptamers candidates, negative selection was performed after the 6th round. The 6th sub-library of ssDNA was incubated with $E$. coli as a counter-partner at $4{ }^{\circ} \mathrm{C}$ for $1 \mathrm{~h}$ in LB broth, and unbound aptamers in the supernatant were collected for the next round of selection.

\subsection{Real-Time PCR Analysis}

To determine the optimal selection round, the eluted ssDNA concentrations from each selection round were analyzed. The ssDNA concentrations from each round were quantified by measuring the absorbance at $260 \mathrm{~nm}$ using a nanodrop spectrophotometer (Thermo Fisher Scientific Korea Ltd., Seoul, Korea). The binding DNA aptamer pool from the initial round, 7 th, 8 th, and 9 th round were prepared using the same concentration (360 pmole) and incubated with target cells $\left(1.2 \times 10^{8} \mathrm{cfu} / \mathrm{mL}\right)$ in parallel for $1 \mathrm{~h}$. After three times washes, bound ssDNA was retrieved from the cell-pool complexes. Serial dilutions of each samples $(1 \times-$ no dilution, $10 \times, 100 \times)$ were prepared and analyzed through real-time PCR using the MiniOpticon ${ }^{\mathrm{TM}}$ Real-time PCR fluorescence signal detection system (Bio-Rad, Hercules, CA, USA). For the amplification, $20 \mu \mathrm{L}$ of reaction mixtures were prepared consisting of $10 \mu \mathrm{L}$ of $1 \mathrm{X}$ iQ SYBR Green Supermix (Bio-Rad), $8.8 \mu \mathrm{L}$ of PCR water, $0.1 \mu \mathrm{L}$ of $0.1 \mathrm{mM}$ of forward primer, $0.1 \mu \mathrm{L}$ of $0.1 \mathrm{mM}$ of reverse primer, and ten-fold serial dilution of each template DNA $(1 \mu \mathrm{L})$. The PCR parameters were: taq activation $\left(3 \mathrm{~min}\right.$ at $\left.94{ }^{\circ} \mathrm{C}\right) ; 40$ cycles of PCR at $94{ }^{\circ} \mathrm{C}$ for $30 \mathrm{~s}$, at $52{ }^{\circ} \mathrm{C}$ for $30 \mathrm{~s}$, and at $72{ }^{\circ} \mathrm{C}$ for $15 \mathrm{~s}$; followed by $5 \mathrm{~min}$ of extension at $72^{\circ} \mathrm{C}$. All sample analyses were performed in triplicate. Efficiency measurement and threshold cycle $(\mathrm{Ct})$ analysis were performed using the $\mathrm{MJ}$ opticon monitor analysis software, version 3.1 (Bio-Rad). The DNA pool from the optimal round (8th round) was cloned into the T-blunt vector in E. coli DH5 $\alpha$ (Solgent, Daejeon, Korea). The purified plasmids from individual white colony were analyzed by sequencing and ClustalX sequence alignment program [30].

\subsection{Post-SELEX for the Binding Affinity Test}

To further confirm the binding affinities of the aptamer candidates to S. sonnei, a post-SELEX step was conducted. A total of 21 aptamer candidates with the same concentration (180 pmole) were prepared and incubated with target cells $\left(1 \times 10^{8} \mathrm{cfu} / \mathrm{mL}\right)$ in parallel. After the post-SELEX process, each aptamer candidates was analyzed through real-time PCR as described above. The quantity of amplicons were analyzed using MiniOpticon ${ }^{\mathrm{TM}}$ Real-time PCR fluorescence signal detection system (Bio-Rad) by comparing the $\mathrm{C}(\mathrm{t})$ values. The secondary structures of the final selected $S$. sonnei binding aptamers were predicted by free energy minimization algorithm using Mfold. (http:/ / mfold.bioinfo. rpi.edu/cgi-bin/dna-form1.cgi). 


\subsection{Binding Activity of Selected Aptamers}

We used the streptavidin immobilized sensor chip SA (for the Biacore 3000, GE, Buckinghamshire, UK) for the interaction test. The 5'-biotin-modified DNA aptamers for S. sonnei (SS-3 and SS-4) were immobilized by passing $500 \mathrm{nM}$ aptamers for $10 \mathrm{~min}$ at $10 \mu \mathrm{L}$ per min on SA chip. The SA chip was pre-activated with $1 \mathrm{~mL}$ of HBS-EP buffer (GE Healthcare, Buckinghamshire, UK) at a flow rate of $10 \mu \mathrm{L}$ per min for $7 \mathrm{~min}$ then $1 \mathrm{~mL}$ of the running buffer at a flow rate of $10 \mu \mathrm{L}$ per min for $10 \mathrm{~min}$. Next, the 5'-biotinylated aptamers were immobilized on the chip as recommended in the manufacturer's manual. To monitor the consecutive interaction, we used the Biacore instrument, and all procedures were automatically implemented to create repetitive cycles of sample injection (90 $\mu \mathrm{L}$ injection samples, at a flow rate of $10 \mu \mathrm{L}$ per min) and regeneration $(1 \mathrm{M} \mathrm{NaCl}, 50 \mathrm{mM}$ $\mathrm{NaOH}, 50 \%$ isopropanol), according to the instruction guidelines (BIA evaluation program, version 4.1, GE Healthcare, Buckinghamshire, UK).

\subsection{Sensitivity Detecting of S. sonnei by Aptamer-Based Fluorescent Biosensor Assay}

Aptamer-based fluorescent biosensor assays were performed using a 96 well microplate with covalently-linked $\mathrm{N}$-oxysuccinimide (NOS) esters that quickly react with primary amine groups (Corning Inc., Corning, NY, USA). In order to varify the aptamer's ability to distinguish S. sonnei from other food-poisoning bacteria (other Shigella species, E. coli, S. typimurium), capture probes and detection probes were chemically synthesized as 5'-amine-modified SS-4 and Cy5-labelled SS-3 at the $5^{\prime}$-end respectively. The assay was prepared as follows: (1) the $5^{\prime}$ amine modified aptamer (SS-4) was coated with a concentration of $1 \mu \mathrm{M}(100 \mathrm{pmol}$ in $100 \mu \mathrm{L}$ treatment volume $)$ per single well NOS (N-oxysuccinimide) surface in a 96-microwell plate for $1 \mathrm{~h}$ at RT. After immobilization, the NOS surfaces were washed 3 times with $0.1 \%$ Tween-20 in TBS (TBST), after which $100 \mu \mathrm{L}$ of blocking buffer ( $2 \%$ BSA in sodium phosphate buffer); (2) To prepare the assay sample, five different bacterial species were spiked into commercially available bottled water with an optical density at $600 \mathrm{~nm}\left(\mathrm{OD}_{600}=0.8\right.$, $\left.4 \times 10^{8} \mathrm{cfu} / \mathrm{mL}\right)$. Next, $100 \mu \mathrm{L}$ of each bacteria samples was added into the wells and incubated with gentle shaking for $1 \mathrm{~h}$ at RT. The unbound cells were removed, and the plates were washed twice with TBST; (3) Finally, $1 \mu \mathrm{M}$ of the Cy5-labeled detecting aptamer (SS-3) was introduced into the individual wells and incubated at RT for $30 \mathrm{~min}$. Following incubation, unbound Cy5 aptamers were washed with TBST, and the absorbance was measured at $650 \mathrm{~nm}$ using the SpectraMax M2 multi-detection microplate reader (Molecular Devices, Sunnyvale, CA, USA) at excitation/emission wavelengths of $646 / 662 \mathrm{~nm}$ respectively.

\section{Conclusions}

In summary, aptamer-based fluorescent biosensor platform to detect $S$. sonnei was developed using a Whole-Bacteria SELEX (WB-SELEX) strategy. We used a post-SELEX procedure and real-time PCR analysis for the highly efficient selection of aptamers. Our platform shows a significant ability to detect and discriminate $S$. sonnei from other enteric species such as E. coli, Salmonella typhimurium and other Shigella species (S. flexneri, S. boydii). The sensitive quantification of $S$. sonnei with a linear dynamic range of $10^{3}-10^{7}$ cells per $\mathrm{mL}$ was also possible using this platform.

Supplementary Materials: Supplementary materials are available online.

Acknowledgments: The authors are grateful for the support of the "Cooperative Research Program for Agriculture Science \& Technology Development (Project No. PJ01191701)" Rural Development Administration, Republic of Korea).

Author Contributions: M.-S.S., S.S.S., and W.-R.S. contributed equally to this work. M.-S.S. and W.-R.S. performed the experiments and analyzed the data; S.S.S., J.M., H.C.K., and W.-R.S. contributed reagents/materials/analysis tools; J.-Y.A. and Y.-H.K. wrote the paper. All authors contributed to the discussion of results and participated in manuscript preparation.

Conflicts of Interest: The authors declare no conflict of interest. 


\section{References}

1. Campilongo, R.; Di Martino, M.L.; Marcocci, L.; Pietrangeli, P.; Leuzzi, A.; Grossi, M.; Casalino, M.; Nicoletti, M.; Micheli, G.; Colonna, B.; et al. Molecular and functional profiling of the polyamine content in enteroinvasive E. coli: Looking into the gap between commensal E. coli and harmful Shigella. PLoS ONE 2014, 9, e106589. [CrossRef] [PubMed]

2. Liu, L.; Oza, S.; Hogan, D.; Perin, J.; Rudan, I.; Lawn, J.E.; Cousens, S.; Mathers, C.; Black, R.E. Global, regional, and national causes of child mortality in 2000-2013, with projections to inform post-2015 priorities: An updated systematic analysis. Lancet 2015, 385, 430-440. [CrossRef]

3. Kotloff, K.L. Bacterial diarrheal pathogens. Adv. Pediatr. Infect. Dis. 1999, 14, 219-267. [PubMed]

4. Lew, J.F.; Swerdlow, D.L.; Dance, M.E.; Griffin, P.M.; Bopp, C.A.; Gillenwater, M.J.; Mercatante, T.; Glass, R.I. An outbreak of shigellosis aboard a cruise ship caused by a multiple-antibiotic-resistant strain of Shigella flexneri. Am. J. Epidemiol. 1991, 134, 413-420. [CrossRef] [PubMed]

5. Gaynor, K.; Park, S.Y.; Kanenaka, R.; Colindres, R.; Mintz, E.; Ram, P.K.; Kitsutani, P.; Nakata, M.; Wedel, S.; Boxrud, D.; et al. International foodborne outbreak of Shigella sonnei infection in airline passengers. Epidemiol. Infect. 2009, 137, 335-341. [CrossRef] [PubMed]

6. Das, S.K.; Chisti, M.J.; Malek, M.A.; Salam, M.A.; Ahmed, T.; Faruque, A.S.; Mondal, D. Comparison of clinical and laboratory characteristics of intestinal amebiasis with shigellosis among patients visiting a large urban diarrheal disease hospital in Bangladesh. Am. J. Trop. Med. Hyg. 2013, 89, 339-344. [CrossRef] [PubMed]

7. Jimenez, K.B.; McCoy, C.B.; Achi, R. Detection of shigella in lettuce by the use of a rapid molecular assay with increased sensitivity. Braz. J. Microbiol. 2010, 41, 993-1000. [CrossRef] [PubMed]

8. Hien, B.T.; Scheutz, F.; Cam, P.D.; Serichantalergs, O.; Huong, T.T.; Thu, T.M.; Dalsgaard, A. Diarrheagenic Escherichia coli and Shigella strains isolated from children in a hospital case-control study in Hanoi, Vietnam. J. Clin. Microbiol. 2008, 46, 996-1004. [CrossRef] [PubMed]

9. Mokhtari, C.; Marchadier, E.; Haim-Boukobza, S.; Jeblaoui, A.; Tesse, S.; Savary, J.; Roque-Afonso, A.M. Comparison of real-time RT-PCR assays for hepatitis E virus RNA detection. J. Clin. Virol. 2013, 58, 36-40. [CrossRef] [PubMed]

10. Sethabutr, O.; Venkatesan, M.; Yam, S.; Pang, L.W.; Smoak, B.L.; Sang, W.K.; Echeverria, P.; Taylor, D.N.; Isenbarger, D.W. Detection of PCR products of the ipaH gene from Shigella and enteroinvasive Escherichia coli by enzyme linked immunosorbent assay. Diagn. Microbiol. Infect. Dis. 2000, 37, 11-16. [CrossRef]

11. Huang, C.J.; Knoll, W.; Sessitsch, A.; Dostalek, J. SPR bacterial pathogen biosensor: The importance of fluidic conditions and probing depth. Talanta 2014, 122, 166-171. [CrossRef] [PubMed]

12. Pierce, S.E.; Bell, R.L.; Hellberg, R.S.; Cheng, C.M.; Chen, K.S.; Williams-Hill, D.M.; Martin, W.B.; Allard, M.W. Detection and Identification of Salmonella enterica, Escherichia coli, and Shigella spp. via PCR-electrospray ionization mass spectrometry: Isolate testing and analysis of food samples. Appl. Environ. Microbiol. 2012, 78, 8403-8411. [CrossRef] [PubMed]

13. Bai, Y.; Feng, F.; Zhao, L.; Wang, C.; Wang, H.; Tian, M.; Qin, J.; Duan, Y.; He, X. Aptamer/thrombin/ aptamer-AuNPs sandwich enhanced surface plasmon resonance sensor for the detection of subnanomolar thrombin. Biosens. Bioelectron. 2013, 47, 265-270. [CrossRef] [PubMed]

14. Liu, J.; Mazumdar, D.; Lu, Y. A simple and sensitive "dipstick" test in serum based on lateral flow separation of aptamer-linked nanostructures. Angew. Chem. Int. Ed. 2006, 45, 7955-7959. [CrossRef] [PubMed]

15. Zhu, Z.; Wu, C.; Liu, H.; Zou, Y.; Zhang, X.; Kang, H.; Yang, C.J.; Tan, W. An aptamer cross-linked hydrogel as a colorimetric platform for visual detection. Angew. Chem. Int. Ed. 2010, 49, 1052-1056. [CrossRef] [PubMed]

16. Sekhon, S.S.; Um, H.J.; Shin, W.R.; Lee, S.H.; Min, J.; Ahn, J.Y.; Kim, Y.H. Aptabody-aptatope interactions in aptablotting Assays. Nanoscale 2017. [CrossRef]

17. Lee, K.A.; Ahn, J.Y.; Lee, S.H.; Singh Sekhon, S.; Kim, D.G.; Min, J.; Kim, Y.H. Aptamer-based sandwich assay and its clinical outlooks for detecting lipocalin-2 in hepatocellular carcinoma (HCC). Sci. Rep. 2015, 5, 10897. [CrossRef] [PubMed]

18. Lee, S.H.; Ahn, J.Y.; Lee, K.A.; Um, H.J.; Sekhon, S.S.; Sun, P.; Min, J.; Kim, Y.H. Analytical bioconjugates, aptamers, enable specific quantitative detection of Listeria monocytogenes. Biosens. Bioelectron. 2015, 68, 272-280. [CrossRef] [PubMed] 
19. Sekhon, S.S.; Kim, S.G.; Lee, S.H.; Jang, A.; Min, J.; Ahn, J.Y.; Kim, Y.H. Advances in pathogen-associated molecules detection using Aptamer based biosensors. Mol. Cell. Toxicol. 2013, 9, 311-317. [CrossRef]

20. Sekhon, S.S.; Lee, S.H.; Lee, K.A.; Min, J.; Lee, B.T.; Kim, K.W.; Ahn, J.Y.; Kim, Y.H. Defining the copper binding aptamotif and aptamer integrated recovery platform (AIRP). Nanoscale 2017, 9, 2883-2894. [CrossRef] [PubMed]

21. Shiao, Y.S.; Chiu, H.H.; Wu, P.H.; Huang, Y.F. Aptamer-Functionalized Gold Nanoparticles As Photoresponsive Nanoplatform for Co-Drug Delivery. ACS Appl. Mater. Interfaces 2014, 6, 21832-21841. [CrossRef] [PubMed]

22. Shieh, Y.A.; Yang, S.J.; Wei, M.F.; Shieh, M.J. Aptamer-based tumor-targeted drug delivery for photodynamic therapy. ACS Nano 2010, 4, 1433-1442. [CrossRef] [PubMed]

23. Gong, W.; Duan, S.; Wu, S.; Huang, Y.; Chen, X.; Wang, Z. Selection, identification, and application of dual DNA aptamers against Shigella sonnei. Anal. Meth. 2015, 7, 3625-3631. [CrossRef]

24. Ahmed, A.M.; Shimamoto, T. Isolation and molecular characterization of Salmonella enterica, Escherichia coli O157:H7 and Shigella spp. from meat and dairy products in Egypt. Int. J. Food Microbiol. 2014, 168, 57-62. [CrossRef] [PubMed]

25. Fellner, L.; Bechtel, N.; Witting, M.A.; Simon, S.; Schmitt-Kopplin, P.; Keim, D.; Scherer, S.; Neuhaus, K. Phenotype of htgA (mbiA), a recently evolved orphan gene of Escherichia coli and Shigella, completely overlapping in antisense to yaaW. FEMS Microbiol. Lett. 2014, 350, 57-64. [CrossRef] [PubMed]

26. Shepherd, J.G.; Wang, L.; Reeves, P.R. Comparison of O-antigen gene clusters of Escherichia coli (Shigella) sonnei and Plesiomonas shigelloides O17: Sonnei gained its current plasmid-borne O-antigen genes from P. shigelloides in a recent event. Infect. Immun. 2000, 68, 6056-6061. [CrossRef] [PubMed]

27. Pupo, G.M.; Lan, R.; Reeves, P.R. Multiple independent origins of Shigella clones of Escherichia coli and convergent evolution of many of their characteristics. Proc. Natl. Acad. Sci. USA 2000, 97, 10567-10572. [CrossRef] [PubMed]

28. Van De Verg, L.L.; Bendiuk, N.O.; Kotloff, K.; Marsh, M.M.; Ruckert, J.L.; Puryear, J.L.; Taylor, D.N.; Hartman, A.B. Cross-reactivity of Shigella flexneri serotype 2a $\mathrm{O}$ antigen antibodies following immunization or infection. Vaccine 1996, 14, 1062-1068. [CrossRef]

29. Baig, I.A.; Moon, J.Y.; Lee, S.C.; Ryoo, S.W.; Yoon, M.Y. Development of ssDNA aptamers as potent inhibitors of Mycobacterium tuberculosis acetohydroxyacid synthase. Biochim. Biophys. Acta 2015, 1854, 1338-1350. [CrossRef] [PubMed]

30. Thompson, J.D.; Gibson, T.J.; Higgins, D.G. Multiple sequence alignment using ClustalW and ClustalX. In Current Protocols in Bioinformatics; Andreas, D.B., Ed.; Wiley: New York, NY, USA, 2002; Chapter 2, Unit 2.3, [CrossRef].

Sample Availability: Samples of the compounds are not available from the authors.

(C) 2017 by the authors. Licensee MDPI, Basel, Switzerland. This article is an open access article distributed under the terms and conditions of the Creative Commons Attribution (CC BY) license (http://creativecommons.org/licenses/by/4.0/). 